\title{
Induction chemoradiotherapy followed by surgery for locally advanced non-small cell lung cancer
}

\author{
SOJIRO KUSUMOTO ${ }^{1}$, TAKASHI HIROSE ${ }^{1}$, MOTOKO FUKAYAMA ${ }^{2}$, DAISUKE KATAOKA ${ }^{2}$, \\ KENJI HAMADA ${ }^{3}$, TOMOHIDE SUGIYAMA ${ }^{1}$, TAKAO SHIRAI ${ }^{1}$, TOSHIMITSU YAMAOKA ${ }^{1}$, \\ KENTARO OKUDA ${ }^{1}$, TSUKASA OHNISHI ${ }^{1}$, TOHRU OHMORI ${ }^{4}$, \\ MITSUTAKA KADOKURA ${ }^{2}$ and MITSURU ADACHI ${ }^{1}$
}

${ }^{1}$ Division of Allergy and Respiratory Medicine, Department of Internal Medicine, ${ }^{2}$ Division of Thoracic Surgery, ${ }^{3}$ Department of Radiology and ${ }^{4}$ Institute of Molecular Oncology, Showa University School of Medicine, 1-5-8 Hatanodai, Shinagawa, Tokyo 142-8666, Japan

Received March 11, 2009; Accepted June 3, 2009

DOI: 10.3892/or_00000549

\begin{abstract}
We examined the efficacy and toxicity of a divided schedule of cisplatin and vinorelbine with concurrent radiotherapy followed by surgery in patients with locally advanced non-small cell lung cancer (NSCLC). Patients with clinical stage IIIA or IIIB NSCLC were eligible if they had a performance status of 0 or 1 , were 75 years or younger, and had adequate organ function. Patients were treated with cisplatin $\left(40 \mathrm{mg} / \mathrm{m}^{2}\right)$ and vinorelbine $\left(20 \mathrm{mg} / \mathrm{m}^{2}\right)$ on days 1 and 8 every 3 weeks. Thoracic radiotherapy ( 2 Gy per fraction; total dose, $40 \mathrm{~Gy}$ ) was given concurrently. Surgical resection was performed after induction therapy had been completed. If disease was considered clinically inoperable after induction therapy, patients received 2 additional cycles of the chemotherapy and 20 Gy of additional radiotherapy. Twenty-three patients (20 men and 3 women; median age, 63 years; age range, 45-72 years) were enrolled. The overall response rate was $78.3 \%$. Although grade 3-4 toxicities included neutropenia in $95.7 \%$ of patients and anemia in $39.1 \%$, no grade $3-4$ radiation pneumonitis or esophagitis occurred. Thirteen patients $(56.5 \%)$ underwent thoracotomy and complete resection. There were no treatment-related deaths. The median survival time was 36 months (range, 4-78 months), the 2-year survival rate was $74 \%$, and the median time to disease progression was 15 months (range, 2-59 months). This trimodality therapy is effective and well tolerated and is an acceptable therapeutic option for patients with locally advanced NSCLC.
\end{abstract}

Correspondence to: Dr Takashi Hirose, Division of Allergy and Respiratory Medicine, Department of Internal Medicine, Showa University School of Medicine, 1-5-8 Hatanodai, Shinagawa, Tokyo 142-8666, Japan

E-mail: thirose-shw@umin.ac.jp

Key words: induction chemoradiotherapy, surgery, non-small cell lung cancer, cisplatin, vinorelbine

\section{Introduction}

Approximately $35 \%$ of patients with non-small cell lung cancer (NSCLC) have locally advanced disease at the time of diagnosis (1). Although surgery is an effective treatment for localized NSCLC, the 5-year survival for patients with clinical stage III disease is less than $15 \%$ (2). Thoracic radio-therapy is also used to treat localized NSCLC, but outcomes remain poor, with median survival times (MSTs) of 9-13 months and 5 -year survival rates of 5-9\% (3). Thus, several combinedtreatment modalities have been used in an attempt to decrease the high rates of local recurrence and distant metastasis.

For patients with a low tumor burden, considered resectable at diagnosis, the aim of induction therapy is to optimize distant disease control by administering chemotherapy at the time of the lowest micrometastatic burden. Several small, randomized trials have shown that survival is longer with induction chemotherapy followed by surgery than with surgery alone $(4,5)$. In addition, a recent meta-analysis has shown a significant survival benefit of $6 \%$ at 5 years for induction chemotherapy followed by surgery over surgery alone (6). On the other hand, Depierre et al found no significant survival benefit for induction chemotherapy and surgery over surgery alone, although they did find a significant survival benefit for the subset of patients with stage IB or II disease (7).

On the other hands, for patients with more extensive metastasis to mediastinal lymph nodes or local invasion, chemotherapy may not be a sufficient induction treatment. Thus, some phase II studies have examined induction chemoradiotherapy followed by surgery for patients with stage IIIA or IIIB NSCLC. These studies have shown promising results for patients with locally advanced NSCLC (8-12). However, the Intergroup 0139 trial has found that median progressionfree survival was significantly longer with chemoradiotherapy followed by surgery than with chemoradiotherapy alone for patients with pathologically proven N2 stage IIIA but that overall survival did not differ (13). Thus, induction chemotherapy or chemoradiotherapy followed by surgery is controversial in patients with locally advanced NSCLC. 
The combination of cisplatin and vinorelbine has become a new standard regimen for metastatic NSCLC (14). In addition, preclinical studies have shown that both cisplatin and vinorelbine act as radiation enhancers. Cisplatin enhances the cytotoxic effects of radiation against tumor cells both in vitro and in vivo (15). Recent studies have shown that vinorelbine enhances the antitumor effects of radiation in vitro in a cell-cycle-dependent manner, with maximal effects when the cells are in the G2/M phase (16).

To date, no phase II trial of a divided schedule of cisplatin and vinorelbine with concurrent radiotherapy followed by surgery in patients with locally advanced NSCLC has been published. Therefore, we performed a phase II study of the combination of a divided schedule of cisplatin and vinorelbine with concurrent radiotherapy followed by surgery in patients with locally advanced NSCLC. The primary end-point of this study was the response rate. The secondary end-points were to investigate survival and toxicity.

\section{Patients and methods}

Eligibility criteria. The criteria for study entry were: i) histologically or cytologically confirmed NSCLC; ii) clinical stage IIIA or IIIB disease; iii) patient age of 20-75 years; iv) Eastern Cooperative Oncology Group performance status of 0 or 1 ; v) measurable lesion; vi) life expectancy of $\geq 3$ months; vii) adequate bone marrow function (leukocyte count of $\geq 4000 / \mu 1$, neutrophil count of $\geq 2000 / \mu 1$, platelet count of $\geq 100000 / \mu \mathrm{l}$, and hemoglobin level of $\geq 9.0 \mathrm{~g} / \mathrm{dl}$ ), renal function (serum creatinine level $<1.5 \mathrm{mg} / \mathrm{dl}$ and creatinine clearance level of $\geq 60 \mathrm{ml} / \mathrm{min}$ ), and hepatic function (total serum bilirubin level less than the upper limit of the normal range, levels of alanine aminotransferase and asparatate aminotransferase less than or equal to twice the upper limits of the normal ranges), and $\mathrm{PaO}_{2}$ of $\geq 70 \mathrm{~mm} \mathrm{Hg}$. Patients had to have a forced expiratory volume in $1 \mathrm{sec}>1.01$. Clinical stage IIIB patients with supraclaviclar lymph node swelling, contralateral hilar lymph node swelling, pleural effusion or pericardial effusion were excluded. In addition, patients were excluded if they had active infections, severe heart disease, interstitial pneumonia, peripheral neuropathy or an active second malignancy. Written informed consent was obtained from all patients.

Treatment protocol. Induction therapy consisted of 2 cycles of cisplatin and vinorelbine with concurrent thoracic radiotherapy. Both cisplatin $\left(40 \mathrm{mg} / \mathrm{m}^{2}\right)$ and vinorelbine $\left(20 \mathrm{mg} / \mathrm{m}^{2}\right)$ were administered on days 1 and 8 . This chemotherapy regimen was repeated every 3 weeks. Thoracic radiotherapy with a total dose of 40 Gy was delivered in 20 fractions starting on day 2. Surgical resection was performed from 3 to 6 weeks after induction therapy had been completed. If patients were considered to have clinically inoperable disease after induction therapy, they received 2 additional cycles of the same chemotherapy regimen and $20 \mathrm{~Gy}$ of additional radiotherapy.

Chemotherapy was discontinued if the treatment outcome was progressive disease or if intolerable toxicity developed at any time. Full doses of cisplatin and vinorelbine were given if the white blood cell (WBC) count was $>2000 / \mu 1$ or if the platelet count was $>75000 / \mu 1$ on day 8 of treatment. If the WBC count was $<3000 / \mu 1$ or if the platelet count was $<100000 /$ $\mu 1$ on day 22 , the second course of chemotherapy was withheld until the count recovered. Cisplatin was permanently discontinued at any time when the serum creatinine level was $>2.0 \mathrm{mg} / \mathrm{dl}$. If the serum creatinine level was $1.5-$ $2.0 \mathrm{mg} / \mathrm{dl}$, cisplatin was withheld for 2 weeks. Prophylactic antiemetic medications were administered to all patients. If the WBC or neutrophil count decreased to more than grade 4 after chemotherapy, granulocyte colony stimulating factor (G-CSF) was administered until the neutrophil count returned to $>1000 / \mu 1$. The doses of both cisplatin and vinorelbine were reduced by $25 \%$ for grade 4 leukopenia or neutropenia lasting 3 days or longer, neutropenic fever during grade 4 neutropenia or grade 4 thrombocytopenia. Chemotherapy was discontinued for grade 3 or higher non-hematologic toxicity, except for alopecia, nausea/vomiting, fever and esophagitis. Radiotherapy was suspended for grade 3-4 esophagitis, fever $>38^{\circ} \mathrm{C}$, grade 4 neutropenia or thrombocytopenia $<20000 / \mu 1$ and was resumed when these toxicities had decreased to grade $\leq 2$. Radiotherapy was discontinued for grade 3-4 radiation pneumonitis.

Radiotherapy was administered to the original volume that had been determined with the size and location of the primary tumor and the draining lymphatic vessels and included a $2-\mathrm{cm}$ margin around the pretreatment primary tumor and the ipsilateral hilum. The entire width of the mediastinum was included, with a $2-\mathrm{cm}$ margin around the radiographically visible area of involvement. The inferior margin extended $3 \mathrm{~cm}$ below the carina or $2 \mathrm{~cm}$ below the radiographically visible tumor mass. When no tumor was detected with physical or radiographic examination in the supraclavicular fossa, this area was not irradiated. The planned initial radiation field was not to exceed $50 \%$ of one lung. The original volume was treated with an anteriorposterior parallel-opposed pair of portals, and the boost volume was treated with the same pair or with a pair of oblique fields if the cumulative radiation dose to the spinal cord exceeded 40 Gy.

For the surgical procedure, lobectomy was preferred; however, bilobectomy or pneumonectomy was performed in cases of primary tumor invasion or regional lymph node metastasis. When necessary, adjacent structures were resected. Unilateral mediastinal lymph node dissection was performed with all pulmonary resections.

Toxicity and evaluation of response. Pretreatment evaluation included a baseline history and physical examination, complete blood cell count with differential, and routine chemistry profiles, urinalysis, chest radiography, chest and abdominal computed tomography (CT), bronchoscopy, magnetic resonance imaging or CT of the brain, electrocardiography, and radionucleotide bone scans. Mediastinoscopy was not included in the staging or restaging work-up. Metastatic lymph nodes were defined as mediastinal lymph nodes $>10 \mathrm{~mm}$ along the short axis on CT scan. After induction therapy had been completed, restaging was performed with the same means used for entry into this study.

Acute toxicities were assessed and graded according to the National Cancer Institute Common Toxicity Criteria 
Table I. Patient characteristics.

\begin{tabular}{lc}
\hline Total no. of patients & 23 \\
Sex (M/F) & $20 / 3$ \\
Mean age (range), years & $63(45-72)$ \\
Performance status (0/1) & $5 / 18$ \\
Histologic type & \\
Adenocarcinoma & 12 \\
Squamous & 11 \\
Stage (IIIA/IIIB) & $18 / 5$ \\
T3N1M0 & 4 \\
T2N2M0 & 11 \\
T3N2M0 & 3 \\
T4N0M0 & 1 \\
T4N1M0 & 1 \\
T4N2M0 & 3 \\
\hline
\end{tabular}

version 2.0, and late toxicity associated with thoracic radiotherapy, occurring $>90$ days after the start of radiotherapy, was graded according to Radiation Therapy Oncology Group late-toxicity criteria. Tumor response was classified according to Response Evaluation Criteria in Solid Tumors criteria. All patients who had received at least 1 cycle of chemotherapy were assessable for response, toxicity and survival.

Statistical considerations. The trial was designed as a phase II study, with response rate as the primary end-point. According to the Simons minimax design, our study, with a sample size of 23 , had $90 \%$ power to accept the hypothesis that the true response rate was $>80 \%$ and $5 \%$ significance to reject the hypothesis that the true response rate was $<50 \%$. Time to progression was defined as the period from the start of this treatment to the date of disease progression or death. Survival time was measured from the start of the present treatment until death or last follow-up. The Kaplan-Meier method was used to calculate survival curves.

\section{Results}

Patients characteristics. From February 2002 through November 2008, 23 patients were enrolled, and all patients could be assessed for survival, response and toxicity (Table I). The patients were 20 men and 3 women with a median age of 63 years (range, 45-72 years). Eighteen patients had stage IIIA disease and 5 patients had stage IIIB disease.

Induction chemoradiotherapy. Of the 23 patients, 18 (78.3\%) achieved a partial response, $4(17.4 \%)$ had stable disease, and $1(4.3 \%)$ had progressive disease, for an overall response rate of $78.3 \%$ (95\% confidence interval, 56.3-92.5\%; Table II). Three $(13.0 \%)$ patients achieved almost pathologically complete response. Disease stage was decreased to stage I in 8 patients and to stage II in 1 patient. The downstaging rate was $39.1 \%$ for all patients and $64.3 \%$ for patients undergoing resection.
Table II. Response rate and types of resection.

\begin{tabular}{lc}
\hline Response rate & $18(78.3 \%)$ \\
Complete response & 0 \\
Partial response & 18 \\
Stable disease & 4 \\
Progressive disease & 1 \\
Resected & $13(56.5 \%)$ \\
Lobectomy & 7 \\
Bi-lobectomy & 2 \\
Pneumonectomy & 4 \\
Right & 1 \\
Left & 3 \\
\hline
\end{tabular}

Doses of both cisplatin and vinorelbine were not reduced in any of the patients who received both 2 courses of chemotherapy and thoracic radiotherapy of $40 \mathrm{~Gy}$. However, doses of both agents were reduced in 2 patients who received 4 courses of chemotherapy and thoracic radiotherapy of $60 \mathrm{~Gy}$, because of grade 4 neutropenia lasting 3 days in 1 patient and neutro-penic fever during grade 4 neutropenia in 1 patient. During the 62 courses of chemotherapy, doses of cisplatin and vinorelbine were skipped once $(1.6 \%)$ on day 8 , because of bacterial pneumonia. Chemotherapy was delayed in 14 courses (23.0\%), usually because of neutropenia. All patients were able to complete radiotherapy by the modification of schedule according to the protocol. Radiotherapy had to be suspended in 4 patients $(17.3 \%)$, because of neutropenia in 3 patients and neutropenic fever in 1 patient.

Toxicity of induction chemoradiotherapy. A total of 62 courses of chemotherapy were given. Table III lists the maximum toxicities occurring during induction chemoradiotherapy. The most frequent toxicity was myelosuppression. Of the 23 patients, $22(95.7 \%)$ had grade 3-4 neutropenia. G-CSF was given during $45.2 \%$ of the courses ( 28 of 62 courses; median duration of administration, 4 days; range, 1-9 days). No patients had grade 3 or 4 thrombocytopenia or hemorrhage. Grade 3-4 anemia occurred in $39.1 \%$ of patients (9 of 23 patients), but none had severe complications related to anemia. Although 19 patients $(82.6 \%)$ had grade 1 or 2 radiation esophagitis, no patients had grade 3 radiation esophagitis. One patient (4.3\%) had grade 2 radiation pneumonitis, but no patients had grade 3-4 radiation pneumonitis. Grade 3 febrile neutropenia occurred in $34.8 \%$ of patients ( 8 of 23 patients), but all patients recovered rapidly after receiving antibiotics.

Surgery. Of the 23 patients, $13(56.5 \%)$ underwent thoracotomy. The initial disease stage in these 13 patients was stage IIIA disease in 11 patients and stage IIIB in 2 patients. The remaining 10 patients did not undergo surgery because of multiple bulky mediastinal lymph node metastases in 3 patients, invasion to important organs in 4 patients, the impossibility of pneumonectomy because of pulmonary or cardiac dysfunction in 2 patients, and progressive disease with multiple bone metastases in 1 patient. Radical resection 
Table III. Toxicity.

\begin{tabular}{lccccc}
\hline & \multicolumn{5}{c}{ National Cancer Institute-Common Toxicity Criteria grade } \\
\cline { 2 - 6 } Toxicity & 1 & 2 & 3 & 4 & 3 or $4(\%)$ \\
\hline Leukopenia & 0 & 1 & 11 & 11 & 95.7 \\
Neutropenia & 0 & 1 & 13 & 9 & 95.7 \\
Thrombocytopenia & 15 & 0 & 0 & 0 & 0 \\
Anemia & 6 & 6 & 7 & 2 & 39.1 \\
Nausea & 11 & 4 & 0 & 0 & 0 \\
Vomiting & 1 & 1 & 0 & 0 & 0 \\
Diarrhea & 3 & 0 & 0 & 0 & 34.8 \\
Infection & 0 & 1 & 8 & 0 & 0 \\
Esophagitis & 15 & 4 & 0 & 0 & 0 \\
Pneumonitis & 0 & 1 & 0 & 0 & 0 \\
Constipation & 10 & 3 & 0 & 0 & 0 \\
Elevation of serum creatinine & 2 & 1 & 0 & 0 & 0 \\
Elevation of transaminase & 6 & 1 & 0 & 0 & 0 \\
Neurologic peripheral & 2 & 0 & 0 & 0 & 0 \\
\hline
\end{tabular}

Survival

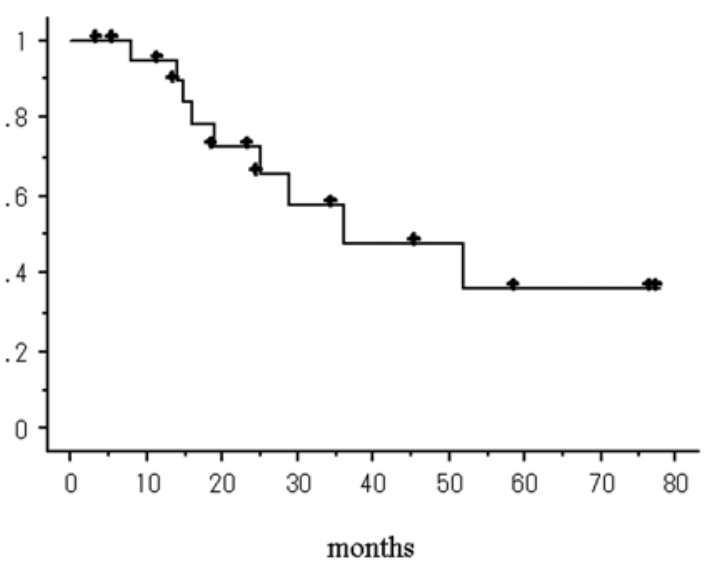

Figure 1. Overall survival time. The median survival time from the start of this regimen was 36 months (range, 4-78 months). Overall survival rates at 2 and 3 years were 74 and $50 \%$, respectively.

was possible in all patients who underwent thoracotomy and included lobectomy in 7 patients, bilobectomy in 2 patients, and pneumonectomy in 4 patients (Table II). Postoperative morbidity rate was $46.2 \%$, including 2 patients with bacterial pneumonia, 1 with liver injury, 2 with hemorrhage from the area of surgery, and 1 with frequent aspiration due to impaired elimination of sputum. There were no treatment-related deaths.

Survival and recurrence. Survival was analyzed when the median follow-up time of all evaluable patients was 21 months. At the time of analysis, 12 patients $(52.2 \%)$ were alive and 2 patients $(8.7 \%)$ had been lost to follow-up. One patient died of bacterial sepsis during second-line chemotherapy for disease recurrence. One patient died of bacterial pneumonia without disease recurrence 52 months after surgery. One
Survival

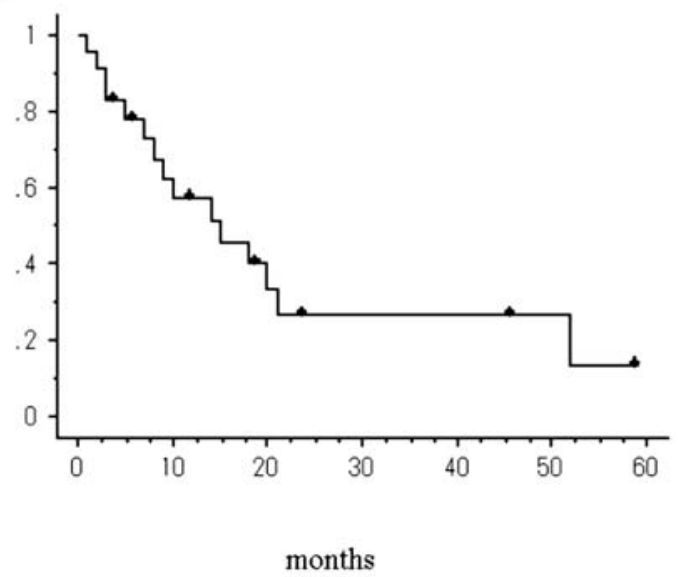

Figure 2. Progression-free survival time. The median time to progression was 15 months (range, 2-59 months).

patient died of colon cancer without disease recurrence 27 months after surgery. The MST from the start of this regimen was 36 months (range, 4-78 months; Fig. 1). Overall survival rates at 2 and 3 years were 74 and $50 \%$, respectively. The MST was not reached in resected patients, whereas it was 25 months in patients who did not undergo surgery $(\mathrm{p}=0.003)$. The median time to progression was 15 months (range, 2-59 months; Fig. 2). The median time to progression was 21 months in resected patients, whereas it was 7 months in patients who did not undergo surgery $(\mathrm{p}=0.04)$.

Of the 23 patients, $14(60.9 \%)$ had recurrent disease. Of the 13 patients undergoing thoracotomy, 6 (46.2\%) had recurrent disease. The sites of recurrence were distant in 5 patients and local in 1 patient, who had recurrence in mediastinal lymph nodes that had not been irradiated. Of the 
10 patients who did not undergo thoracotomy, $8(80 \%)$ had recurrent disease. The sites of recurrence were local in 4 patients, distant in 2 patients, and both local and distant in 2 patients. The rate of recurrence was slightly but not significantly higher in patients not undergoing thoracotomy than in patients undergoing thoracotomy $(\mathrm{p}=0.10)$. The sites of distant metastasis (some patients had recurrent disease at more than 1 site) were the lungs in 4 patients, the brain in 2 patients, bone in 2 patients, the liver in 1 patient, and the adrenal gland in 1 patient.

\section{Discussion}

Although surgery is an effective treatment for localized NSCLC, the 5-year survival for patients with clinical stage III disease is less than $15 \%$ (2). Several combined treatment modalities have been used in an attempt to decrease the high rates of local recurrence and distant metastasis (17). Some phase II studies of induction chemoradiotherapy followed by surgery have obtained promising results for patients with locally advanced NSCLC (8-12). However, there is no evidence that induction chemoradiotherapy followed by surgery is superior to chemoradiotherapy or surgery alone. In addition, a standard induction chemoradiotherapy regimen, including radiation dose and schedule, choice of chemotherapeutic agents, and sequence of chemotherapy and radiotherapy, has not been determined. Thus, research is needed to develop induction chemoradiotherapy regimens that are less toxic and more effective.

In the present study, the response rate to induction chemoradiotherapy was $78.3 \%$, the rate of complete resection was $58.5 \%$, the MST was 36 months, and the survival rate at 2 years was $74 \%$. These results compare favorably with those of previous trials of trimodality therapy in patients with locally advanced NSCLC, although comparing these results with those of other studies is difficult because of the inclusion of patients with different prognostic factors (8-12). In all of these studies, the response rates to induction chemoradiotherapy have ranged from 59 to $81 \%$, the complete surgical resection rates have ranged from 45 to $81 \%$, MSTs have ranged from 13 to 25 months, and survival rates at 2 years have ranged from 26 to $66 \%$ (8-12). Additionally, previous studies have reported that downstaging is correlated with rates of survival and distant recurrence $(12,18)$. In previous studies, the rate of downstaging has ranged from 12 to $33 \%$ $(12,13)$. In the present study, the rate of downstaging was $39.1 \%$ among all patients and $64.3 \%$ among patients undergoing thoracotomy.

A previous study has reported that induction chemoradiotherapy is associated with a significantly higher incidence of major postoperative complications than is induction chemotherapy alone (19). Surgical mortality rates after induction chemoradiotherapy have ranged from 4 to $11 \%(8-12,20)$. In the present study, there were no treatment-related deaths. One possible reason for the absence of treatment-related deaths is that only 1 patient underwent right sided pneumonectomy. Pneumonectomy is a major risk factor for mortality, especially for patients with right-sided tumors (21). The Intergroup 0139 trial reported the $26 \%$ operative mortality rate in patients undergoing pneumonectomy (13). In addition, no patients had grade 3 to 4 radiation pneumonitis or esophagitis. In patients who were to receive surgery, radiotherapy was stopped at $40 \mathrm{~Gy}$, according to the recommendation of International Association for the Study of Lung Cancer consensus report that the dose of $45 \mathrm{~Gy}$ of radiotherapy not be exceeded at conventional fractions before surgery (10). This approach could explain the low rate of non-hematologic toxicities and the absence of treatment-related deaths.

Until now, the potential role of a surgical resection following chemotherapy with or without radiotherapy has been controversial in patients with locally advanced NSCLC. Meerbeeck et al have reported that surgical resection does not improve overall or progression-free survival compared with radiotherapy in patients with pathologically proven stage IIIA-N2 disease that has responded to induction chemotherapy (20). Thus, Meerbeeck et al have suggested that chemotherapy followed by radiotherapy should be considered the preferred treatment for patients with pathologically proven stage IIIA-N2 (20). However, there remain high rates of both locoregional and systemic failures in chemoradiotherapy in patients with stage III NSCLC. It is hypothesized that by resecting the residual disease after chemoradiotherapy, a proportion of these patients might be rendered disease-free. Therefore, the addition of surgical resection to chemoradiotherapy is a potential option for improving local control.

Recently, cisplatin-based adjuvant chemotherapy has been standard therapy in patients with stage II or III NSCLC $(22,23)$. A pooled analysis of large adjuvant trials of cisplatinbased chemotherapy for NSCLC has shown that adjuvant chemotherapy significantly improves survival (5.4\% absolute benefit at 5 years) over surgery alone in patients with stage II or III NSCLC (22). The optimal sequence of chemotherapy with respect to surgery (induction or adjuvant) has yet to be determined in patients with stage III NSCLC. Compared to adjuvant chemotherapy, induction therapy is often better tolerated, resulting in a higher rate of treatment compliance. Additionally, induction therapy may eliminate potential micrometastatic disease at the earliest possible time. On the other hands, potential drawbacks of induction therapy are delayed surgical resection and less accurate staging. We believe that rather than induction therapy and adjuvant therapy being competing therapies, they might be complementary therapies that could be used for different subgroups of patients. Induction therapy might be best used when tumors need downstaging preoperatively. Further large prospective study is needed to determine optimal approach for stage III NSCLC.

Our study had at least one limitation. Staging was not as accurate as it might have been, because mediastinoscopy was not included in the staging or restaging work-up. In the present study, metastatic lymph nodes were defined as mediastinal lymph nodes larger than $10 \mathrm{~mm}$ along the short axis on CT scan. However, in the diagnosis of mediastinal lymph node metastasis with CT scan, with a sensitivity of $40-75 \%$ and a specificity of $40-66 \%$, is inferior to mediastinoscopy, with a sensitivity of $80 \%$ to $90 \%$ and a specificity of $100 \%$, or to positron-emission tomography, with a sensitivity of $90 \%$ and a specificity of $86 \%$ (24-26). Additionally, the exact stage of T3 or T4 tumors is difficult to determine with a CT scan. The sensitivity and specificity of CT scan for 
distinguishing T3 or T4 tumors from T0 or T2 tumors is 63 and $84 \%$, respectively (27).

In conclusion, the present phase II study is the first to examine the combination of a divided schedule of cisplatin and vinorelbine with concurrent radiotherapy followed by surgery for locally advanced NSCLC. Although survival data are preliminary because of the short follow-up time, the overall response rate was $78.3 \%$ with MST of 36 months and a survival rate at 2 years of $74 \%$. Furthermore, neither severe toxicity during induction chemoradiotherapy nor severe operative morbidity or mortality occurred. Therefore, this trimodality therapy could be an effective treatment and an acceptable option for patients with stage III NSCLC when tumors need downstaging preoperatively.

\section{References}

1. Jemal A, Tiwari RC, Murray T, et al: American Cancer Society: Cancer Statistics, 2004. CA Cancer J Clin 54: 8-29, 2004.

2. Mountain CF: Revisions in the international system for staging lung cancer. Chest 111: 1710-1717, 1997.

3. Perez CA, Stanley K, Grundy G, et al: Impact of irradiation technique and tumor extent in tumor control and survival of patients with unresectable non-oat cell carcinoma of the lung. Cancer 50: 1091-1099, 1982.

4. Rosell R, Gómez-Codina J, Camps C, et al: A randomized trial comparing preoperative chemotherapy plus surgery with surgery alone in patients with non-small cell lung cancer. N Engl J Med 330: 153-158, 1994

5. Roth JA, Fossella F, Komaki R, et al: A randomized trial comparing perioperative chemotherapy and surgery with surgery alone in resectable stage IIIA non-small cell lung cancer. J Natl Cancer Inst 86: 673-680, 1994

6. Burdett S, Stewart LA and Rydzewska L: A systematic review and meta-analysis of the literature: chemotherapy and surgery versus surgery alone in non-small cell lung cancer. J Thorac Oncol 1: 611-621, 2006

7. Depierre A, Milleron B, Moro-Sibilot D, et al: French Thoracic Cooperative Group: preoperative chemotherapy followed by surgery compared with primary surgery in resectable stage I (except T1N0), II and IIIA non-small cell lung cancer. J Clin Oncol 20: 247-253, 2002

8. Albain KS, Rusch VW, Crowley JJ, et al: Concurrent cisplatin/ etoposide plus chest radiotherapy followed by surgery for stage IIIA (N2) and IIIB non-small cell lung cancer: mature results of Southwest Oncology Group phase II study 8805 . J Clin Oncol 13: $1880-1892,1995$.

9. Choi NC, Carey RW, Daly W, et al: Potential impact on survival of improved tumor downstaging and resection rate by preoperative twice-daily radiation and concurrent chemotherapy in stage IIIA non-small cell lung cancer. J Clin Oncol 15: 712-722, 1997.

10. Katakami N, Okazaki M, Nishiuchi S, et al: Induction chemoradiotherapy for advanced stage III non-small cell lung cancer: long-term follow-up in 42 patients. Lung Cancer 22: 127-137, 1998

11. Eberhardt W, Wilke H, Stamatis G, et al: Preoperative chemotherapy followed by concurrent chemoradiotherapy based on hyperfractionated accelerated radiotherapy and definitive surgery in locally advanced non-small-cell lung cancer: mature results of a phase II trial. J Clin Oncol 16: 622-634, 1998.
12. Trodella L, Granone P, Valente S, et al: Neoadjuvant concurrent radiochemotherapy in locally advanced (IIIA-IIIB) non-small cell lung cancer: long-term results according to downstaging. Ann Oncol 15: 389-398, 2004.

13. Albain KS, Scott CB, Rusch VR, Turrisi AT, Shepherd FA, Smith C for RTOG, SWOG, NCIC, ECOG, CALGB, NCCTG: Phase III comparison of concurrent chemotherapy plus radiotherapy (CT/radiotherapy) and CT/radiotherapy followed by surgical resection for stage IIIA (pN2) non-small cell lung cancer (NSCLC): initial results from Intergroup trial 0139 (RTOG 93-09). Proc Am Soc Clin Oncol 22: 621, 2003.

14. Le Chevalier T, Brisgand D, Douilland JY, et al: Randomized study of vinorelbine and cisplatin versus vindesine and cisplatin versus vinorelbin alone in advanced non-small cell lung cancer: results of a European multicentre trial including 612 patients. J Clin Oncol 12: 360-367, 1994.

15. Dewit L: Combined treatment of radiation and cis-diamminedichloroplatinum II: a review of experimental and clinical data. Int J Radiat Oncol Bio Phys 13: 403-426, 1987.

16. Edelstein MP, Wolfe LA III and Duch DS: Potentiation of radiation therapy by vinorelbine (Navelbine) in non-small cell lung cancer. Semin Oncol 23: 41-47, 1996.

17. Farray D, Mirkovic N and Albain KS: Multimodality therapy for stage III non-small cell lung cancer. J Clin Oncol 23: 3257-3269, 2005.

18. Lorent N, De Leyn P, Lievens Y, et al: Long-term survival of surgically staged IIIA-N2 non-small cell lung cancer treated with surgical combined modality approach: analysis of a 7-year prospective experience. Ann Oncol 15: 1645-1653, 2004.

19. Macchiarini P, Chapelier AR, Monnet I, et al: Extended operations after induction therapy for stage IIIB (T4) non-small cell lung cancer. Ann Thorac Surg 57: 966-973, 1994.

20. Van Meerbeeck JP, Kramer GW, van Schil PE, et al: European Organisation for Research and Treatment of Cancer-Lung Cancer Group: randomized controlled trail of resection versus radiotherapy after induction chemotherapy in stage IIIA-N2 non-small cell lung cancer. J Natl Cancer Inst 99: 442-450, 2007.

21. Martin J, Ginsberg RJ, Abolhoda A, et al: Morbidity and mortality after neoadjuvant therapy for lung cancer: the risks of right pneumonectomy. Ann Thorac Surg 72: 1149-1154, 2001.

22. Pignon JP, Tribodet H, Scagliotti GV, et al: LACE Collaborative Group: Lung adjuvant cisplatin evaluation: a pooled analysis by the LACE Collaborative Group. J Clin Oncol 26: 3552-3559, 2008.

23. The International Adjuvant Lung Cancer Trial Collaborative Group: Cisplatin-based adjuvant chemotherapy in patients with completely resected non-small cell lung cancer. N Eng J Med 350: 351-360, 2004.

24. Steinert HC, Hauser M, Allemann F, Engel H, Berthold T, von Schulthess GK and Weder W: Non-small cell lung cancer: nodal staging with FDG PET versus CT with correlative lymph node mapping and sampling. Radiology 202: 441-446, 1997.

25. Gdeedo A, van Schil P, Corthouts B, van Mieghem F, van Meerbeeck J and van Marck E: Prospective evaluation of computed tomography and mediastinoscopy in mediastinal lymph node staging. Eur Resp J 10: 1547-1551, 1997.

26. Pieterman RM, van Putten JW, Meuzelaar JJ, et al: Preoperative staging of non-small cell lung cancer with positron-emission tomography. N Eng J Med 343: 254-261, 2000.

27. Webb WR, Gatsonis C, Zerhouni EA, Heelan RT, Glazer GM, Francis IR and McNeil BJ: CT and MR imaging in staging non-small cell bronchogenic carcinoma: report of the radiologic diagnostic oncology group. Radiology 178: 705-713, 1991. 